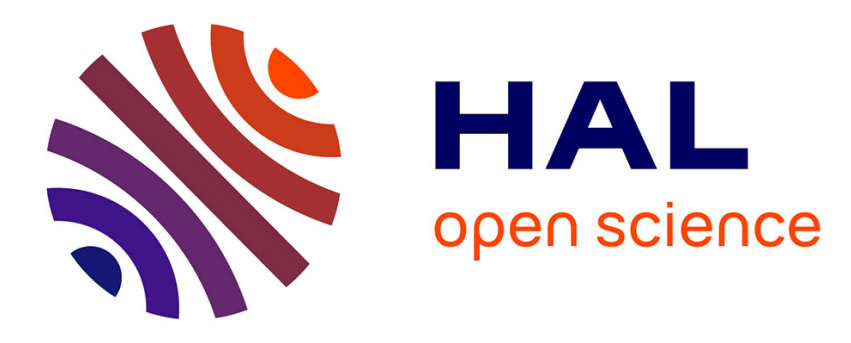

\title{
Modeling a Priori Information on the Velocity Field in Reflection Tomography
}

D. Sinoquet

\section{To cite this version:}

D. Sinoquet. Modeling a Priori Information on the Velocity Field in Reflection Tomography. SEG Annual Meeting, Sep 1993, Washington, United States. hal-02284180

\section{HAL Id: hal-02284180 \\ https://hal-ifp.archives-ouvertes.fr/hal-02284180}

Submitted on 11 Sep 2019

HAL is a multi-disciplinary open access archive for the deposit and dissemination of scientific research documents, whether they are published or not. The documents may come from teaching and research institutions in France or abroad, or from public or private research centers.
L'archive ouverte pluridisciplinaire HAL, est destinée au dépôt et à la diffusion de documents scientifiques de niveau recherche, publiés ou non, émanant des établissements d'enseignement et de recherche français ou étrangers, des laboratoires publics ou privés. 


\section{Modeling a Priori Information on the Velocity Field}

in Reflection Tomography

\section{S U M M A R Y}

Reflection tomography consists in determining a velocity field and reflector geometries from traveltimes picked on multioffset seismic section. The solution of the tomographic inverse problem being underdetermined, we need to integrate a priori information on the model. A regularization by means of model curvature has in general no physical justification and leads to geologically incorrect models. This paper presents a formulation that integrates in a realistic way a priori geological information associated with the regularity of the model and with the relation between the velocity distribution and the interface geometries. Such an integration seems particularly critical when dealing with smooth velocity models in which velocity and interfaces are defined independently from each other. We validate the interest of our formulation on a real data example.

\section{INTRODUCTION}

Conventional seismic processing can not image complex geological structures such as the one shown in Figure 1. Lateral velocity variations in the vicinity of the fault prevent complete imaging of the base of salt and of the underlying structure.

Successful seismic imaging of such geologic structures requires prestack depth migration which itself requires the determination of an accurate velocity model.

Reflection tomography (Bishop et al., 1985, Chiu et al., 1987) seems very attractive for this determination. In this method the velocity distribution and the geometry of the interfaces are determined from traveltimes picked on prestack seismic sections.

Because of the finite number of data and of the infinite number of unknowns the tomographic inverse problem is ill posed. To offset the indetermination additional information on the model is necessary. Delprat-Jannaud and Lailly (1993) proposed a regularization by means of the model curvature that insures the uniqueness of the solution and mathematical stability. Unfortunately this method is unstable in practice when applied to complex geologic structures where the curvature of the velocity can, in no way, be understood as smooth. As a consequence of this unstability the velocity distribution and the structural model can be found completly uncorrelated from each other as shown in Figure 2. Even if the model matches the picked traveltimes, it is geologically unacceptable.

More generally, the solution of reflection tomography is basically underdetermined and relevant geologic information has to be integrated to remove this underdetermination. This underdetermination is quite critical in complex structures where difficulties in picking seismic reflections make the traveltime data very sparse. In this paper we propose a method for the integration of some specific geologic information which is particularly suited for applications to complex structures.

\section{REFLECTION TOMOGRAPHY ON COMPLEX GEOLOGIC STRUCTURES SMOOTH VELOCITY MODELS}

We are primarly interested in applications of reflection tomography for the determination of a velocity model to be used as input to prestack depth migration to image complex structures.

Discontinuous velocity models are not suitable for such a migration: misplaced discontinuities in the velocity distribution create discontinuous slopes in the migrated reflections that can completely mislead the interpreter. To preserve the nice features of reflection tomography, namely its ability to produce a velocity model kinematically consistent with the seismic data, the velocity model used for the migration should be identical to the one obtained by tomography. This lead us to build a reflection tomography dealing with smooth velocity models (Figure 2).

\section{TOMOGRAPHY PROBLEM}

Reflection tomography involves the solution of two problems: the forward problem and the inverse problem.

Forward problem:

We deal with two-dimensional media limited to a rectangular domain $\Omega$ defined by a horizontal interval $\left.I_{x}=\right] x_{\min }, x_{\max }[$ and by a depth interval $\left.I_{z}=\right] z_{\text {min }}, z_{\text {max }}[$.

The model $m$ is composed of a slowness squared distribution $u(x, z)$, that is supposed to be $C^{2}(\Omega)$ and of explicit interface depth functions $z_{i}(x)$ supposed to be $C^{2}\left(I_{x}\right), i$ indicating the number of interface $(i=1, \ldots, N)$.

The forward problem is the computation of traveltimes in a given model by raytracing. We consider acoustic waves propagating away from a source, reflecting on an interface (reflector) and propagating to a receiver at the surface. A ray $R_{j}$ is defined by the triple (source, reflector, receiver) indexed by $j$. We define the corresponding traveltime as $T_{j}(m)=\int_{R_{j}} \sqrt{u} d s$.

Inverse problem:

We have at our disposal a set of observed traveltimes $T^{o b s}$ and we look for the model that matches these data at best. The least square formulation of the inverse problem consists in minimizing the misfit between observed and computed traveltimes:

$$
C(m)=\left\|T(m)-T^{o b s}\right\|^{2}
$$

where $\|$.$\| is a norm in data space, for instance the Euclidian$ $\mathrm{n}$ or $\mathrm{m}$.

This non linear inverse problem is solved by the GaussNewton method which consists in solving successive linearized problems that can be expressed as the minimization of the 
functional

$$
\grave{C}\left(\partial m^{n+1}\right)=\left\|J\left(m^{n}\right) \partial m^{n+1}-\partial T^{o b s}\right\|^{2}
$$

with $m^{n}$ a given model, $J\left(m^{n}\right)$ the Jacobian of the forward modelling operator $T(m), \partial T^{o b s}=T^{o b s}-T\left(m^{n}\right), \partial m^{n+1}=$ $m^{n+1}-m^{n}$, a model perturbation giving the new model $m^{n+1}$.

These linearized inverse problems are underdetermined: they admit several solutions. Additional information is necessary to overcome this difficulty.

\section{A PRIORI INFORMATION ON VELOCITY STRUCTURE}

The subsurfaces of interest are not chaotic media: the interpreter knows the sedimentary deposition modes and the unconformity surfaces from regional geology. A certain velocity structure results from such an organization. We use two types of geological information on the velocity field: information on the regularity of the velocity and on the anisotropy of regularity due to sedimentation. (Sinoquet and Brac, 1992)

Regularity of the velocity:

For complex structure tomography, we deal only with macroscopic phenomena: very slight velocity variations will not be determined by traveltime inversion. Versteeg (1991) showed that only velocity field wavelengths greater than 100 meters are of importance for prestack migration. Accordingly, this defines the objective for complex structure tomography. Additionally, geology tells us that the velocity is more regular in some places than in others, even though it is assumed smooth. Figure 3 shows the wide velocity variations in the medium, particularly in the neighborhood of the fault and at the base of the salt, as well as at the hard and soft carbonate contacts (at the right of the fault) and between these carbonates and the clay-sand sequence.

Anisotropy of regularity (influence of sedimentation):

The sedimentation makes regular, roughly horizontal deposits. In turn the velocity varies much less along deposition lines in the orthogonal direction (Figure 4). Therefore the velocity varies little along the reflectors that are geological isochrons.

To model the above described a priori information we add penalization terms to the tomography objective function

$$
\begin{gathered}
\Theta^{\prime}(m)=\underbrace{\left\|T(m)-T^{\text {obs }}\right\|^{2}}_{\text {Tomography }}+\underbrace{\sum_{i=1}^{N} \int_{I_{i}} \varepsilon_{1}^{i}(m, x, z)^{2}\left(\vec{\nabla} u \cdot \vec{t}_{i}\right)^{2} d x}_{\text {velocity guide }} \\
+\underbrace{\int_{\Omega} \varepsilon_{2}(m, x, z)^{2}\left[\left(\frac{\partial^{2} u}{\partial x^{2}}\right)^{2}+\left(\frac{\partial^{2} u}{\partial z^{2}}\right)^{2}+\left(\frac{\partial^{2} u}{\partial x \partial z}\right)^{2}\right] d x d z}_{\text {velocity regularization }} \\
+\underbrace{\sum_{i=1}^{N}\left(\varepsilon_{3}^{i}\right)^{2} \int\left(\frac{\partial^{2} z_{i}}{\partial x^{2}}\right)^{2} d x}_{\text {interface regularization }}
\end{gathered}
$$

where $\vec{\nabla} u$ is the gradient of the slowness squared and $\vec{t}_{i}$ is the unit tangent vector to the interface $I_{i}$.
The velocity guide term constrains the slowness squared (consequently the velocity) to vary little along certain reflectors: it integrates the second type of a priori information. The regularization terms constrain the model to be regular and help to propagate the local velocity guide on the interfaces into the layer. But the velocity regularization term plays also an active role in making the velocity distribution in accordance with the reflector geometries. By imposing gentle variations of the velocity gradient, we make this gradient remain approximately orthogonal to the geological isochrons that are within a layer between the reflectors involved in the application of reflection tomography. Consequently the isovelocity lines and the geological isochrons are expected to have similar directions within a whole layer.

The variable weighting functions $\varepsilon_{k}^{2}$ represent the uncertainty associated with each piece of information. They enable us to calibrate the functional terms according to their physical meaning. They are space varying and depend also on the model to allow for instance possible degenerescence of the velocity regularization in the vicinity of faults whose geometry is an unknown of reflection tomography.

The linearized problem associated with the minimization of the objective function $C^{\prime}(m)$ is a well posed problem: as in Delprat-Jannaud and Lailly (1993) uniqueness and stability of the solution are ensured.

\section{NUMERICAL RESULTS}

\section{NUMERICAL METHOD}

Parameterization of the model:

The model is represented by cubic B spline functions, adequate to build $C^{2}$ functions (Barsky et al., 1987). The velocity parameters are the vertices of a tensor of B spline functions (respectively depending on $x$ and on $z$ ). The interface depths are represented by B spline function depending on $x$.

\section{Optimization:}

We minimize the non quadratic objective function (3) by the Gauss-Newton method. Each linearized problem is solved by a block relaxation method: we update velocity parameters and interface parameters alternatively and we iterate the process. Such a method avoids ill conditionning difficulties involved in the minimization of a function that depends on parameters of different physical natures. At each relaxation step we use an algorithm of constrained optimization based on the Augmented Lagrangian method, developped by Glowenski and Tran (1993). It allows to integrate linear constraints such as imposing an interface slope or imposing the velocity to be smaller than a certain value.

\section{COMPARISON OF MODELS OBTAINED BY TOMOGRAPHY WITH DIFFERENT A PRIORI INFORMATION}

The model in Figure 2 was obtained by tomography with curvature regularization (Delprat-Jannaud and Lailly, 1993), whereas the model in Figure 5 is the result of tomography integrating the a priori information described above. Both models match the traveltime data. 
Basically, models associated with complex structures do not have small curvature. Thus to obtain a model that matches the traveltime data we can only use very weak regularization, thus leading to unstability in pratice (Delprat-Jannaud and Lailly, 1993). Therefore it is not surprising that the model in Figure 2 is geologically unacceptable. Indeed, there is no coherency between the velocity field and the interface geometries. Moreover, as a consequence of the weak curvature regularization, unrealistic velocity anomalies appear: they reflect the underlying unstability.

To obtain the model in Figure 5, we have constrained the velocity to vary little along the interfaces, except along the fault and the base of salt. All the interfaces has been regularized by second derivatives. Also the second derivatives of the velocity are penalized everywhere but with different weights according to the region. Indeed, we define three zones: the neighborhood of the fault, where the regularization weight is very low to allow wide velocity variations, and the two regions on both sides of the fault.

All the unrealistic features of the model in Figure 2 have disappeared in the model in Figure 5:

- the velocity varies regularly within each layer

- the isovelocity lines follow nicely the interfaces.

In the neighborhood of the fault, the velocity is not well determined because of the weak regularization introduced in this area.

So, we obtained a model which matches the traveltimes and which is geologically acceptable. But as we will see in the next section, a difficulty remains to obtain such a model: the choice of the weights associated with the a priori information.

\section{INCIDENCE OF THE CHOICE OF WEIGHTS}

The model in Figure 6 was obtained in the same way as the model in Figure 5 but with different weights: we increased weights associated with the velocity regularization and with the velocity guide.

This model is very regular on both sides of the fault and presents high velocity variations at the top and the bottom of the fault. The model respects the a priori information but does not match traveltimes. This means that the information we try to integrate is not consistent. Therefore a good compromise has to be found between the conflictual pieces of information and the choice of weights has to be adequate.

In fact, we have to choose their values according to the physical uncertainties they represent. This is far from obvious a priori. However, an iterative trial and error approach turned out to be quite constructive to calibrate those weights. If the weights guessed a priori have led to the model in Figure 6 we conclude that, the traveltime match being unacceptable, we should increase the associated weight or, equivalently, decrease the weights associated with the velocity guide or with the velocity curvature or both. This trial and error approach was effectively used to calibrate iteratively the weights so as to obtain the model in Figure 5.

\section{CON CLUSION}

Integration of a priori geological information especially on the smoothness of the subsurface is crucial in reflection tomography: it is the warrant of stability.

Integrating information ensuring coherency between the velocity distribution and geometry of the interfaces is essential when tomography makes use of a velocity distribution represented by a continuous function. Such a representation is appropriate when the velocity computed by reflection tomography is to be used as input to prestack depth migration for the imaging of complex structures. Thus we proposed a modeling of the regional regularity of the velocity and of the sedimentation effects on its structure.

Solving a tomography problem integrating such a priori information leads to the minimization of a multi-objective function. A weight is associated with each term of the functional. These weights have been model space varying to allow degenerescence of the velocity regularization in the vicinity of faults, for example. The tuning of these weights is quite critical because it defines the compromise to choose between conflictual pieces of information.

A correct tuning has been obtained by a trial and error approach, thus leading to a model matching both traveltime data and the a priori geologic information.

\section{ACKNOWLEDGMENTS}

This research was carried out as part of the Prestack Structural Interpretation consortium project (PSI). I hereby acknowledge the support provided by the sponsors of this project.

I also wish to thank Patrick Lailly, Jean Brac and Florence Delprat-Jannaud for their valuable advice. I want to thank Elf Aquitaine for its financial support.

\section{REF E REN CES}

Barsky, R.H., Bartels, B.A. and Beatty, J.C., 1986, An introduction to splines for use in computer graphics and geometry modeling, Morgan Kaufmann Publishers

Bishop, T.N., Bube, K.P., Cutler, R.T., Love, P.L., Resnick, J.R., Shuey, R.T., Spindler, D.A., and Wyld, H. W., 1985, Tomographic determination of velocity and depth in laterally varying media, Geophysics, vol.50, 903-923

Chiu, S.K.L., and Stewart, R.R., 1987, Tomographic determination of three-dimensionnal seismic velocity structure using well logs, vertical seismic profiles, and surface seismic data, Geophysics, vol. 52, 10854098

Delprat-Jannaud, F. and Lailly, P., 1993, Ill posed and well posed formulations of the reflection tomography problem, accepted for publication in J.G.R.

Sinoquet, D. and Brac, J., 1992, Modeling a priori information on velocity field for reflection tomography, PSI 1992 Annual Report, Institut Francais du Petrole, Rueil, France

Glowinski, R. and Tran, Q.H., 1993, Constrained optimization in reflection tomography: the Augmented Lagrangian method, submitted to Journal of optimization, SIAM

Versteeg, R.J., Analyse du probleme de la determination du modele de vitesse pour l'imagerie sismique, these de Doctorat, Universite Paris VII, France 


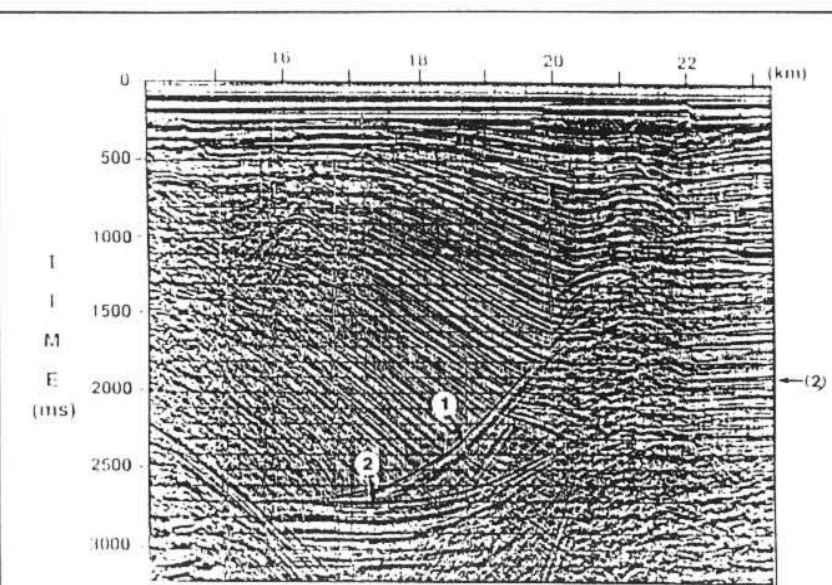

Figure 1 CMP stacked section. 1: Fault, 2: Base of salt.

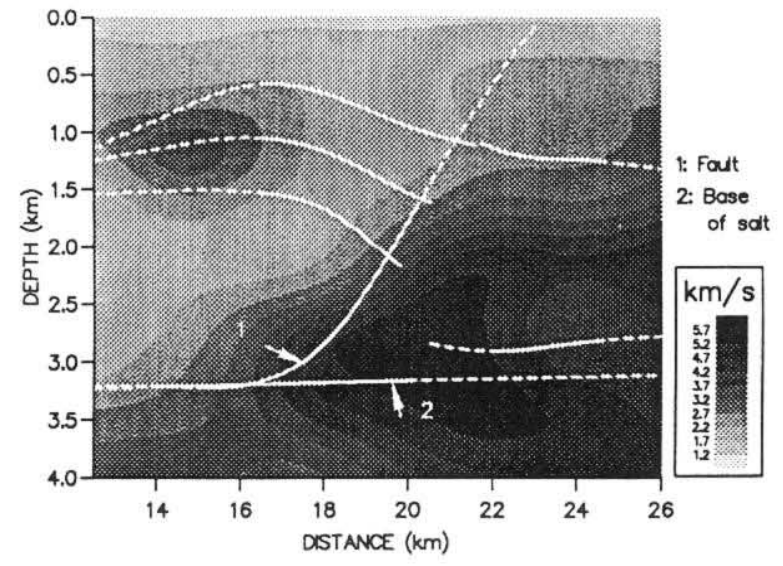

Figure 2 Model obtained by reflection tomography with curvature regularization. This model corresponds to the section in Figure 1. 7 reflected events were picked on seismic sections with offsets ranging from $160 \mathrm{~m}$ to $2500 \mathrm{~m}$. Parts of the interfaces that are not illuminated are shown by doted lines. RMS traveltime misfit: $14.6 \mathrm{~ms}$.

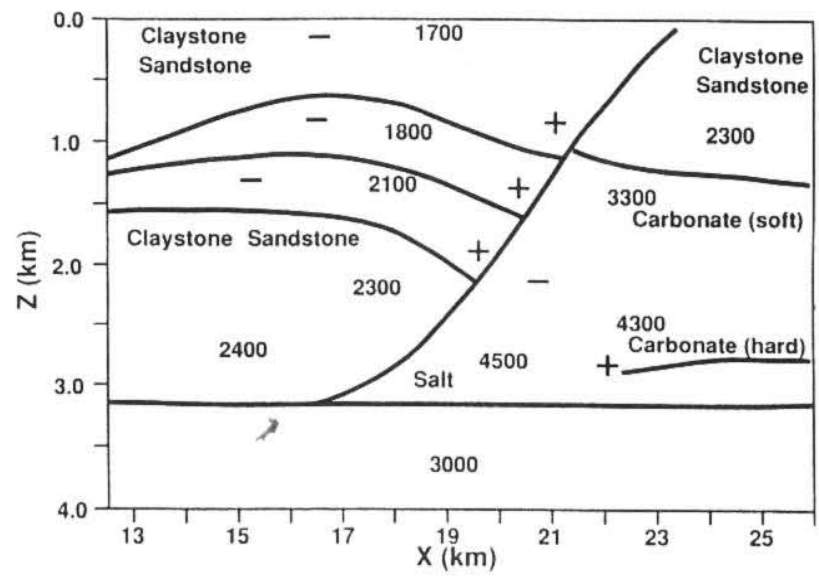

Figure 3 Lithology of the subsurface and possible variations of the velocity field $\left(m . s^{-1}\right),+$ indicates higher velocity, - indicates lower velocity.

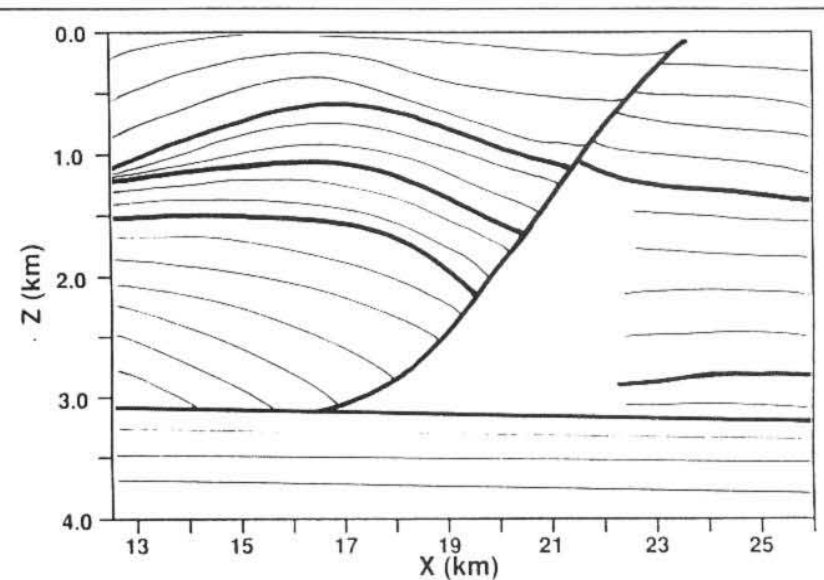

Figure 4 Possible distribution of isovelocity lines according to sedimentation effects.

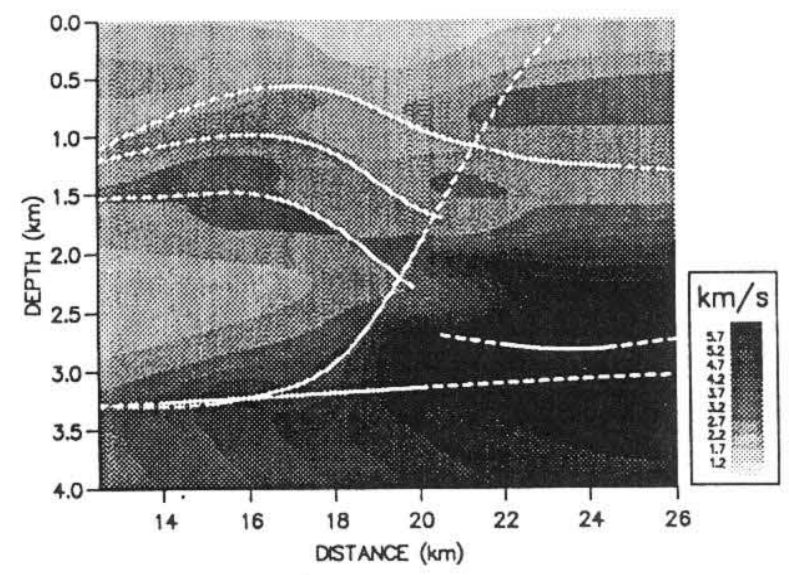

Figure 5 Model obtained by reflection tomography with a priori information on velocity structure with the following weight values: tomography: $\varepsilon_{0}=1$ - velocity guide: $\varepsilon_{1}=5.10^{-1}$ - velocity regularization: $\varepsilon_{2}$ fault region: $10^{-5}$, left and right sides: $10^{-2}-$ interface regularization: $\varepsilon_{3}=10^{-2}$. RMS traveltime misfit : $17 \mathrm{~ms}$.

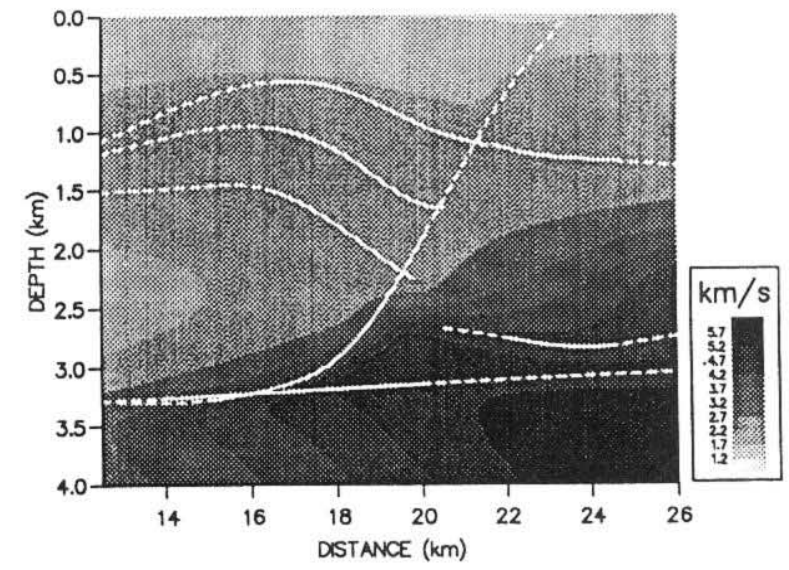

Figure 6 Model obtained by reflection tomography with a priori information on velocity structure but with a different choice of the weight values: tomography: $\varepsilon_{0}=1$ - velocity guide: $\varepsilon_{1}=10$ - velocity regularization: $\varepsilon_{2}$ fault region: $10^{-5}$, left and right sides: 1 - interface regularization: $s_{3}=10^{-2}$. Average traveltime misfit : $112 \mathrm{~ms}$. 